\title{
Phonons and Quantum Criticality Revealed by Temperature Linear Resistivity in Twisted Double Bilayer Graphene
}

\section{Yanbang Chu}

Beijing National Laboratory for Condensed Matter Physics and Institute of Physics, Chinese Academy of Sciences

\section{Le Liu}

Beijing National Laboratory for Condensed Matter Physics; Key Laboratory for Nanoscale Physics and Devices, Institute of Physics, Chinese Academy of Sciences, Beijing 100190

\section{Cheng Shen}

Beijing National Laboratory for Condensed Matter Physics; Key Laboratory for Nanoscale Physics and Devices, Institute of Physics, Chinese Academy of Sciences, Beijing 100190

Jinpeng Tian

Institute of Physics, Chinese Academy of Sciences

Jian Tang

Institute of Physics, The Chinese Academy of Sciences https://orcid.org/0000-0003-1324-2466

\section{Yanchong Zhao}

Beijing National Laboratory for Condensed Matter Physics and Institute of Physics, Chinese Academy of Sciences https://orcid.org/0000-0002-5472-8828

\section{Jieying Liu}

Beijing National Laboratory for Condensed Matter Physics and Institute of Physics, Chinese Academy of

Sciences

\section{Yalong Yuan}

Beijing National Laboratory for Condensed Matter Physics; Key Laboratory for Nanoscale Physics and Devices, Institute of Physics, Chinese Academy of Sciences, Beijing 100190

Yiru Ji

Beijing National Laboratory for Condensed Matter Physics; Key Laboratory for Nanoscale Physics and Devices, Institute of Physics, Chinese Academy of Sciences, Beijing 100190

\section{Rong Yang}

Beijing National Laboratory for Condensed Matter Physics; Key Laboratory for Nanoscale Physics and Devices, Institute of Physics, Chinese Academy of Sciences, Beijing 100190 https://orcid.org/00000003-2216-7584

\section{Kenji Watanabe}

National Institute for Materials Science https://orcid.org/0000-0003-3701-8119 


\section{Takashi Taniguchi}

National Institute for Materials Science, Tsukuba, Ibaraki https://orcid.org/0000-0002-1467-3105

\section{Dongxia Shi}

Institute of Physics, The Chinese Academy of Sciences

\section{Fengcheng Wu}

School of Physics and Technology, Wuhan University, Wuhan 430072, China

\section{Wei Yang ( $\square$ wei.yang@iphy.ac.cn )}

Beijing National Laboratory for Condensed Matter Physics; Key Laboratory for Nanoscale Physics and Devices, Institute of Physics, Chinese Academy of Sciences, Beijing 100190 https://orcid.org/00000002-3925-0352

\section{Guangyu Zhang}

Institute of Physics, Chinese Academy of Sciences https://orcid.org/0000-0002-1242-4391

\section{Article}

Keywords: twisted double bilayer graphene (TDBG), phonon and quantum criticality, physics Posted Date: April 28th, 2021

DOl: https://doi.org/10.21203/rs.3.rs-447319/v1

License: (c) (i) This work is licensed under a Creative Commons Attribution 4.0 International License. Read Full License 


\title{
Phonons and Quantum Criticality Revealed by Temperature Linear Resistivity in Twisted Double Bilayer Graphene
}

Yanbang $\mathrm{Chu}^{1,2}$, Le Liu ${ }^{1,2}$, Cheng Shen ${ }^{1,2}$, Jinpeng Tian ${ }^{1,2}$, Jian Tang ${ }^{1,2}$, Yanchong Zhao ${ }^{1,2}$, Jieying Liu ${ }^{1,2}$, Yalong Yuan ${ }^{1,2}$, Yiru $\mathrm{Ji}^{1,2}$, Rong Yang ${ }^{1,3,4}$, Kenji Watanabe ${ }^{5}$, Takashi Taniguchi ${ }^{6}$, Dongxia $\mathrm{Shi}^{1,2,3,4}$, Fengcheng $\mathrm{Wu}^{7}$, Wei Yang ${ }^{1,2,3,4^{*}}$, Guangyu Zhang $^{1,2,3,4^{*}}$

${ }^{1}$ Beijing National Laboratory for Condensed Matter Physics and Institute of Physics, Chinese Academy of Sciences, Beijing 100190, China

${ }^{2}$ School of Physical Sciences, University of Chinese Academy of Sciences, Beijing, 100190, China

${ }^{3}$ Beijing Key Laboratory for Nanomaterials and Nanodevices, Beijing 100190, China

${ }^{4}$ Songshan Lake Materials Laboratory, Dongguan, Guangdong, 523808, China

${ }^{5}$ Research Center for Functional Materials, National Institute for Materials Science, 1-1 Namiki, Tsukuba 305-0044, Japan

${ }^{6}$ International Center for Materials Nanoarchitectonics, National Institute for Materials Science, 1-1 Namiki, Tsukuba 305-0044, Japan

${ }^{7}$ School of Physics and Technology, Wuhan University, Wuhan 430072, China

*Corresponding authors: wei.yang@iphy.ac.cn; gyzhang@iphy.ac.cn

\begin{abstract}
Twisted double bilayer graphene (TDBG) is an electric-field-tunable moiré system, exhibiting electron correlated states and related temperature linear (T-linear) resistivity. The displacement field provides a new knob to in-situ tune the relative strength of electron interactions in TDBG, yielding not only a rich phase diagram but also the ability to investigate each phase individually. Here, we report a study of carrier density $(n)$, displacement field $(D)$ and twist angle $(\theta)$ dependence of T-linear resistivity in TDBG. For a large twist angle $\left(\theta>1.5^{\circ}\right)$ where correlated insulating states are absent, we observe a T-linear resistivity (order of $10 \Omega / \mathrm{K}$ ) over a wide range of carrier density and its slope decreases with increasing of $n$ before reaching the van Hove singularity, in agreement with acoustic phonon scattering model. The slope of T-linear resistivity is non-monotonically dependent on displacement field, with a single peak structure closely connected to single-particle van Hove Singularity (vHS) in TDBG. For an optimal twist angle of $\sim 1.23^{\circ}$ in the presence of correlated states, the slope of T-linear resistivity is found maximum at the boundary of the correlated halo regime (order of $100 \Omega / \mathrm{K}$ ), resulting a ' $\mathrm{M}$ ' shape displacement field dependence. The observation is beyond the phonon scattering model from single particle picture, and instead it suggests a strange metal behavior. We interpret the observation as a result of symmetry-breaking
\end{abstract}


instability developed at quantum critical points where electron degeneracy changes. Our results demonstrate that TDBG is an ideal system to study the interplay between phonon and quantum criticality, and might help to map out the evolution of the order parameters for the ground states.

\section{Main Text}

Resistance is a measure of how electrons are transported and scattered in solids, and it gives rich and fundamental information of the given system. It usually shows a power law dependence on temperature (T), $\rho \propto T^{\alpha}$, where $\alpha$ is a coefficient that differs from one scattering mechanism to another. Of particular interest is linear regime when $\alpha=1$, since it could originate from conventional acoustic phonon couplings (Fig. 1a) ${ }^{[1]}$, but also it might indicate unconventional processes that rooted in quantum criticality ${ }^{[2-4]}$ (Fig. 1b). The latter strange metal behaviors are observed in a wide range of correlated systems, for instance cuprates ${ }^{[5]}$, iron-based compounds ${ }^{[6,7]}$, heavy fermion systems ${ }^{[8,9]}$, Kondo lattices ${ }^{[10]}$, and frustrated lattices ${ }^{[11,12]}$, where quantum critical points are found at the instabilities of order parameters. So far, most studies focus on individual states and are limited by in-situ sample tunability, research on the critical behavior near critical points is still lacking yet.

Twisted moiré superlattice has emerged as a new platform for hosting rich correlated states. In twisted bilayer graphene (TBG), the generation of low energy flat bands ${ }^{[13]}$ quenches kinetic energy of electrons and brings about correlated insulating state $^{[14]}$, superconductivity ${ }^{[15,16]}$, ferromagnetism ${ }^{[17,18]}$. The superconducting dome ${ }^{[15,16]}$ and temperature linear (T-linear) resistivity ${ }^{[19,20]}$ above critical temperature in TBG bears a lot of similarity with that in optimally doped cuprates ${ }^{[20,21]}$. In twisted double bilayer graphene (TDBG), another graphene-based twisted 2D material system, observations of spin-polarized correlated insulating state, superconducting-like states and T-linear resistivity have been reported ${ }^{[22-24]}$. Compared to TBG, TDBG is more advantaged in tuning electron interactions, aside from twist angle. This is achieved by in-situ tuning of displacement field, which changes the flat bandwidth $\mathrm{W}$ and band separation $\Delta$ in the band structure of $\mathrm{TDBG}^{[25]}$. The displacement field acts as an extra free parameter to control the relative strength of electron interactions to kinetic energy, not only contributing a versatile phase diagram, but also allowing an in-situ switch between different phases in TDBG, avoiding variation between different samples. Meanwhile, with temperature scaling of resistance as the probe, one can elucidate the dominating scattering mechanism, and then estimate the relative strength of electronphonon coupling or electron interaction at low temperature where correlated states emerge and switch ${ }^{[19,26]}$

In this work, we systematically study T-linear resistivity in TDBG. We found Tlinear resistivity in a wide range of twist angle, from 'optimal' twist angle $1.23^{\circ}$ to relatively larger twist angle $1.91^{\circ}$. We first start with a large twist angle device where correlated insulating states are absent, and find the dominating role played by acoustic phonons for the T-linear resistivity. Meanwhile, we also demonstrate a displacement 
field tunable electron-phonon interaction and its intimate connection with van Hove singularity (vHS). And then in the optimal twist angle devices, we reveal and highlight the features beyond the phonon model, and discuss the role played by quantum criticality in TDBG.

The TDBG samples are prepared by 'cut and stack' technique ${ }^{[27]}$, encapsulated by top and bottom hBN flakes, and then they are deposited on $\mathrm{SiO}_{2} / \mathrm{Si}$ substrate. The devices are designed with a dual gate Hall bar geometry and are fabricated in bubblefree region of the samples (see Methods for details). The dual gate geometry enables us to tune carrier density (n) and electric displacement field (D) independently, via carrier density $n=\left(C_{B G} V_{B G}+C_{T G} V_{T G}\right) / e$ and displacement field $D=\left(C_{B G} V_{B G}-\right.$ $\left.C_{T G} V_{T G}\right) / 2$, where $C_{B G}$ and $C_{T G}$ are the geometrical capacitance per area for bottom and top dielectric layer, $V_{B G}$ and $V_{T G}$ are the bottom and top gate voltage, and $e$ is the elementary charge. All our devices have length-to-width ratios close to one, with a twist angle $\theta$ of $1.23^{\circ}, 1.33^{\circ}, 1.42^{\circ}, 1.55^{\circ}$ and $1.91^{\circ}$.

Figure 2a show the resistivity as a function of $n$ and $D$ at a temperature of $1.7 \mathrm{~K}$ in $1.55^{\circ}$ device. There are three prominent resistance peaks, corresponding to charge neutral point (CNP) and superlattice gaps on both electron $\left(+n_{s}\right)$ and hole $\left(-n_{s}\right)$ branches, as labeled in Fig.2a. Here, $n_{s}=4 / A \approx 8 \theta^{2} / \sqrt{3} a^{2}$ is the carrier density at full filling of a moiré unit cell and from which twist angle $\theta$ can be determined, where $a$ is the lattice constant of graphene and a prefactor of 4 comes from the valley and spin degeneracy in TDBG mini-Brillouin zone. For an optimal twist angle of $1.23^{\circ}$, correlated insulating states (CS) are observed at half filling of conduction moiré subband and surrounded by halo structure in Fig.3a, consistent with previous works ${ }^{[22-}$ ${ }^{24]}$. While for $1.55^{\circ}$, the correlated behavior is absent even though the displacement field is widely tuned, as shown in Fig.2a. Continuum model gives the displacement field tunable band structure of TDBG (Fig. S15 and S16), qualitatively consistent with experimental observations. We observe T-linear resistivity in a wide range of twist angle, $n$, and $D / \varepsilon_{0}$, as shown in Fig. S1-S5, Fig. 2b-c and Fig. 3b-c. It's worth noting that the linear slope $\mathrm{dR} / \mathrm{dT}$ changes with $n, D$, and $\theta$.

We first focus on devices in which correlated states are absent at low temperature. Figure $2 \mathrm{~d}$ shows the extracted $\mathrm{dR} / \mathrm{dT}$ as a function of moiré filling factor $v$ in $1.55^{\circ}$ and $1.91^{\circ}$ devices, where $v$ is the number of carriers in one moiré unit cell defined by $v=$ $4 n / n_{s}$. In both devices, $\mathrm{dR} / \mathrm{dT}$ decreases with increasing $v$, despite of the overall smaller $\mathrm{dR} / \mathrm{dT}$ in $1.91^{\circ}$ device. Normalized $\mathrm{dR} / \mathrm{dT}$ shows similar $v$ dependence (Fig. $\mathrm{S} 13$ ) in these two devices, suggesting the same origin of T-linear resistivity.

We attribute the T-linear resistivity in these devices to the electron-phonon interactions in TDBG. According to a theoretical calculation of electron-phonon scattering in $\mathrm{TDBG}^{[28]}$, acoustic phonon scattering is enhanced by renormalized Fermi velocity in TDBG moiré subband. And it predicts a similar crossover from $\mathrm{T}$ at high temperature to $\mathrm{T}^{4}$ at low temperature $(\mathrm{T}<10 \mathrm{~K})$. If one take electron-electron Umklapp scattering ${ }^{[29]}$, it is more likely $\mathrm{T}^{2}$ when phonons occupation number are vanishing at 
low temperature. In the linear regime, the $\mathrm{dR} / \mathrm{dT}$ resulting from acoustic phonon scattering is given by ${ }^{[28]}$

$$
d R / d T=\frac{\pi D^{2} k_{B} z_{\infty}}{2 g_{s} g_{v} e^{2} \hbar \rho_{m} v_{p h}^{2} v_{F}^{2}}
$$

where $g_{s}, g_{v}$ are spin and valley degeneracies $\rho_{m}$ is the mass density, $D$ is acoustic phonon deformation potential, $v_{p h}$ and $v_{F}$ are phonon velocity and Fermi velocity, $z_{\infty}$ is the integral concerning phonon occupation and other scattering details in TDBG.

This model predicts a strong carrier density dependence for a parabolic dispersion in $2 \mathrm{D}$ electron system, which yields $v_{F} \sim k_{F}$ and thus results in $d R / d T \propto 1 / n$. In our experiments, we observed a linear dependence of $\mathrm{dR} / \mathrm{dT}$ over $1 / \mathrm{n}$ for $1.55^{\circ}$ devices at $D / \varepsilon_{0}=0 \mathrm{~V} / \mathrm{nm}$ in Fig. S6c and for $1.23^{\circ}$ device in Fig. S6e-g. Note that a change of Fermi surface topology would lead to a deviation from $1 / \mathrm{n}$ dependence. For instance, $\mathrm{dR} / \mathrm{dT}$ is found linear with $1 / \sqrt{n}$ for $1.55^{\circ}$ devices at $D / \varepsilon_{0}=-0.45 \mathrm{~V} / \mathrm{nm}$ as well as $1.91^{\circ}$ devices at $D / \varepsilon_{0}=0$ and $-0.4 \mathrm{~V} / \mathrm{nm}$ in Fig. S6a-b, d. This contrast might indicate a renormalized parabolic band in TDBG, leading to $v_{F}^{2} \propto \sqrt{n}$ under certain conditions. Besides, van Hove Singularity (vHS) can also change the Fermi surface topology and results in a deviation of $\mathrm{dR} / \mathrm{dT}$ from $1 / \mathrm{n}$ dependence. The vHS by continuum model ${ }^{[23]}$ in TDBG shows up as a cross-like feature in the phase diagram of $R(n, D)$, indicated by the black dashed lines in Fig.2f and Fig.S12. The upturn of $\mathrm{dR} / \mathrm{dT}$ at around $v=2$ for $1.55^{\circ}$ device at $D / \varepsilon_{0}=-0.45 \mathrm{~V} / \mathrm{nm}$ (Fig. $2 \mathrm{~d}$ ) is consistent with the weak cross-like feature. In both cases, the T-linear resistance can be well accounted by acoustic phonon scattering, and we name it 'conventional metal' (M) here.

The above arguments are further supported in the strongly displacement field tunable T-linear resistivity. Figure $2 \mathrm{e}$ shows $\mathrm{dR} / \mathrm{dT}$ as a function of $D / \varepsilon 0$ at different $v$ in $1.55^{\circ}$ device, and clearly it shows a non-monotonical and strong dependence that peaks on finite $D / \varepsilon_{0}$. As indicated by the arrow in Fig. 2e, the peak position (yellow dots) in $D / \varepsilon_{0}$ (D_peak) shifts with moiré band filling factor $v$ linearly, in parallel with resistance peak (black dashed line) in Fig. $2 \mathrm{f}$. The resistance peak is a reminiscent of the cross-like feature due to vHS in band structure, which might point to a close connection between T-linear resistivity to the vHS. The field dependent $\mathrm{dR} / \mathrm{dT}$ suggests a field tunable Fermi velocity or more fundamentally a tunable moiré band topology in TDBG, in agreement with the calculated field tunable moiré bandwidth (Fig. S15). However, there remains one puzzle regarding the offset between yellow dots and the black dashed lines, as the band should be flattest at vHS and thus contribute a dR/dT peak according to the acoustic phonon scenario.

Next, we turn to the $1.23^{\circ}$ device in Fig. 3a, and the carrier density dependence of $\mathrm{dR} / \mathrm{dT}$ (Fig. S5 and S6) in general agrees with the acoustic phonon contribution. Here, we focus on the field dependence of $\mathrm{dR} / \mathrm{dT}$ in Fig. $4 \mathrm{a}$. Similar to the results from $1.55^{\circ}$ and $1.91^{\circ}$ devices, the $\mathrm{dR} / \mathrm{dT}$ is strongly field tunable yet with a much larger magnitude. Moreover, the previous single peak in Fig. 2e develops into two in Fig. 4a as the doping tuned close to half filling $\mathrm{v}=2$. A new peak emerges at a lower field and eventually 
results in ' $M$ ' shaped displacement field dependence at half filling $v=2$ (Fig. 4a and Fig.S7). The intensity of the new peak quickly diminished away from half filling (i.e. $\mathrm{v}<2$, see Fig. S7 and 8), leaving only shoulder-like feature. Surprisingly, these peaks are found following the boundary of the halo structure in Fig. 4c. These observations suggest the ' $\mathrm{M}$ ' feature already emerges weakly when TDBG is tuned to the halo but strongly enhanced near $\mathrm{v}=2$. The same behaviors are also observed in $1.33^{\circ}$ device (Fig. S4d).

The " $\mathrm{M}$ " shaped field dependence of $\mathrm{dR} / \mathrm{dT}$ at half filling $\mathrm{v}=2$ is strongly linked with the emergence of correlated insulating states. We plot the $\mathrm{dR} / \mathrm{dT}$ and $\mathrm{R}$ together in Fig. 3b, and it's intriguing to find that the two peaks of the ' $M$ ' feature locate exactly at the field where correlated insulating states start to emerge. As the twist angle is increased to $1.42^{\circ}$ (Fig. S11, middle panel), where correlated insulating states are not well developed, a shoulder-like feature of two peaks is still visible. It totally disappears at $1.55^{\circ}$ (Fig. S11, bottom panel), where no signature of CS are found. However, the valley of " $\mathrm{M}$ " shaped $\mathrm{dR} / \mathrm{dT}$ is unlikely related to the correlated gap opening, since it persists in the metallic halo regime.

The peaks that envelop the halo structure are intimately connected to critical points of the spontaneous symmetry breaking. We plot the Hall coefficient, $R_{H a l l}=\left[R_{x y}(+B)-\right.$ $\left.\mathrm{R}_{\mathrm{xy}}(-\mathrm{B})\right] / 2 \mathrm{~B}$, together with $\mathrm{dR} / \mathrm{dT}$ in Fig. $4 \mathrm{~d}$ and Fig.S9. The $\mathrm{dR} / \mathrm{dT}$ peaks are found concurrent with a sharp change of $\mathrm{R}_{\text {Hall. }}$. The sharp change of Hall coefficient demonstrates the critical point between symmetry broken state and conventional metal, in agreement with the observations in literature ${ }^{[30]}$.

Note that the insulator at half filling and the halo structure are strongly connected with the cross-like vHS features. If we extend the crossed lines from the hole band to the electron band, as indicated by the black dashed lines in Fig. 3a for the $1.23^{\circ}$ device, the vHS are found penetrating through the halo structure and correlated insulator in the center. At the half filling, the resistance is maximum at round $\mathrm{D}=-0.35 \mathrm{~V} / \mathrm{nm}$ in Fig. $4 \mathrm{~b}$, coinciding with the field where single particle vHS are expected, however, the $\mathrm{dR} / \mathrm{dT}$ is found minimum there, deviating from acoustic phonon model. In short, the carrier density dependence at a fixed displacement field of the $1.23^{\circ}$ device agrees with the phonon model, while the displacement field dependence at a fixed carrier density is beyond the phonon model from single particle picture. This is very similar to the case of the offset between $\mathrm{R}$ and $\mathrm{dR} / \mathrm{dT}$ at larger twist angles where correlated insulators absent in Fig. 2f.

We attribute the anomalous $\mathrm{dR} / \mathrm{dT}$ peaks in displacement field at a fixed carrier density to a consequence of symmetry breaking at the critical points between different phases. At these critical points, quantum fluctuations are generated as a result of the competing between different phases (ground states). In TDBG, it is 4-fold degenerate outside the halo structure while it is 2 -fold or even non-degenerate inside ${ }^{[30]}$. The boundary of the halo structure separate states with different symmetry, and is likely to bring about a symmetry-breaking instability. These fluctuations could drive the T- 
linear resistivity all the way from ground state at low temperature up to high temperature where all these fragile and delicate ground states disappeared. Similar to the strange metal in cuprate or frustrated lattices, the charge instability orfluctuation leads to an enhanced electron-phonon interaction near critical points ${ }^{[11]}$. In addition, we suspect that the strange offset between $R$ and $d R / d T$ for $1.55^{\circ}$ device in Fig. $2 \mathrm{f}$ where correlated insulating states absent is of the same origin. Conversely, that is to say the strange metal behavior in Fig. 2 f suggests a more subtle and possible symmetrybroken ground states buried at a lower temperature. Indeed, signatures of halo and correlated insulating states are observed at a slightly smaller twist angle of 1.53 degree in Ref. 23. Thus, one would expect the T-linear resistivity at high temperature provides information of ground states at low temperature. However, more investigations are expected in the future for a better understanding experimentally and theoretically.

The quantum criticality picture is better captured at critical points in the phase diagram when $\mathrm{T}<10 \mathrm{~K}$. Fig. $3 \mathrm{~d}(3 \mathrm{e})$ show a mapping of resistivity to temperature and displacement field for $1.23^{\circ}$ device at $v=2$ (1.8), with corresponding numerical derivative dR/dT shown in Fig. $3 \mathrm{f}(3 \mathrm{~g})$. The critical points of the halo regime, regardless of correlated insulating or metallic states, are marked by black arrows in Fig. 3d-g. It is vividly shown in Fig. $3 \mathrm{f}$ and $3 \mathrm{~g}$ that there is a change from superconducting like or $\mathrm{T}^{2}$ to $\mathrm{T}$ when displacement field is approaching to critical points, a generation of quantum critical points by electrical field. At the critical points, the high temperature linear slope $\mathrm{dR} / \mathrm{dT}$ is maximized, and moreover the T-linear resistivity could persist down to a low temperature well below the $\mathrm{T}_{\mathrm{BG}}$ (marked by red stars Fig. 3b-c). Such a T-linear resistivity approaching the base temperature is a characteristic quantum critical behavior.

In conclusion, we systematically investigate the carrier density, displacement field and twist angle dependences of T-linear resistivity in TDBG. We demonstrate a dominant role played by acoustic phonons when correlated states are absent. The Tlinear resistivity has a non-monotonic displacement field dependence with a single $\mathrm{dR} / \mathrm{dT}$ peaks related to $\mathrm{vHS}$, revealing the field-tunable electron phonon interaction in TDBG. Moreover, we find features that beyond the phonon model in the presence of correlated states near optimal twist angle. Instead of a single peak, there is a second $\mathrm{dR} / \mathrm{dT}$ peak at lower displacement field and thus it results in ' $\mathrm{M}$ ' shaped displacement field dependence. These peaks are found located at the boundary of the halo structure, separating states with different symmetry. We attribute the unconventional $\mathrm{dR} / \mathrm{dT}$ peaks to a consequence of fluctuations due to quantum criticality. Our observations establish a close link between high temperature T-linear resistivity to low temperature ground states, and would inspire more works about the nature of quantum criticality and ground states instability in TDBG. And similar analysis may also work in other field-tunable systems such as twisted monolayer-bilayer graphene ${ }^{[31-33]}$ and $\mathrm{ABC}$ stacking trilayer graphene/hBN moiré system ${ }^{[34]}$. 


\section{Acknowledgement}

We thank Rui Zhou, Lede Xian, Xiao Li for helpful discussion. This work was supported by the National Key Research and Development Program (Grant No. 2020YFA0309604), the NSFC (Grants Nos. 61888102, 11834017, and 12074413), the Strategic Priority Research Program of CAS (grant Nos.XDB30000000 and XDB33000000), the Key-Area Research and Development Program of Guangdong Province (Grant No.2020B0101340001), and the Research Program of Beijing Academy of Quantum Information Sciences under Grant No. Y18G11. Growth of hexagonal boron nitride crystals was supported by the Elemental Strategy Initiative conducted by the MEXT, Japan, Grant Number JPMXP0112101001, JSPS KAKENHI Grant Number JP20H00354 and A3 Foresight by JSPS.

\section{Author contribution}

Y.C., C.S., W.Y., and G.Z. conceived the project. Y.C., L.L. and C.S. fabricated the devices and performed the transport measurements. Y.C., L.L. and J.T. provided the samples. L.L. provided continuum model calculations. K.W. and T.T. provided hexagonal boron nitride crystals. Y.C., W.Y., and G.Z. analyzed the data. Y.C. and W.Y. wrote the paper. All authors discussed and commented on this work.

\section{Reference}

1 Ziman, J. M. Electrons and phonons: the theory of transport phenomena in solids. (Oxford university press, 2001).

2 Varma, C. M. Colloquium: Linear in temperature resistivity and associated mysteries including high temperature superconductivity. Rev. Mod. Phys. 92, 031001, (2020).

3 Coleman, P. \& Schofield, A. J. Quantum criticality. Nature (London) 433, 226-229, (2005).

4 Sachdev, S. \& Keimer, B. Quantum criticality. Phys. Today 64, 29, (2011).

5 Keimer, B., Kivelson, S. A., Norman, M. R., Uchida, S. \& Zaanen, J. From quantum matter to high-temperature superconductivity in copper oxides. Nature (London) 518, 179-186, (2015).

6 Shibauchi, T., Carrington, A. \& Matsuda, Y. A quantum critical point lying beneath the superconducting dome in iron pnictides. Annu. Rev. Condens. Matter Phys. 5, 113-135, (2014).

Zhou, R. et al. Quantum criticality in electron-doped BaFe 2- x Ni x As 2. Nat. Commun.

4, 1-7, (2013).

8 Gegenwart, P., Si, Q. \& Steglich, F. Quantum criticality in heavy-fermion metals. Nat. Phy. 4, 186-197, (2008).

9 Stewart, G. Non-Fermi-liquid behavior in d-and f-electron metals. Rev. Mod. Phys. 73, 797, (2001).

10 Shen, B. et al. Strange-metal behaviour in a pure ferromagnetic Kondo lattice. Nature (London) 579, 51-55, (2020).

11 Cano-Cortes, L., Merino, J. \& Fratini, S. Quantum critical behavior of electrons at the edge 
of charge order. Phys. Rev. Lett. 105, 036405, (2010).

Sato, T. et al. Strange metal from a frustration-driven charge order instability. Nat. Mat. 18, 229-233, (2019).

Bistritzer, R. \& MacDonald, A. H. Moire bands in twisted double-layer graphene. Proc. Natl. Acad. Sci. U.S.A. 108, 12233-12237, (2011).

14 Cao, Y. et al. Correlated insulator behaviour at half-filling in magic-angle graphene superlattices. Nature 556, 80-84, (2018).

15 Cao, Y. et al. Unconventional superconductivity in magic-angle graphene superlattices. Nature 556, 43-50, (2018).

Lu, X. et al. Superconductors, orbital magnets and correlated states in magic-angle bilayer graphene. Nature 574, 653-657, (2019).

Sharpe, A. L. et al. Emergent ferromagnetism near three-quarters filling in twisted bilayer graphene. Science 365, 605-608, (2019).

18 Serlin, M. et al. Intrinsic quantized anomalous Hall effect in a moire heterostructure. Science 367, 900-903, (2020).

Polshyn, H. et al. Large linear-in-temperature resistivity in twisted bilayer graphene. Nat. Phy. 15, 1011-1016, (2019).

Cao, Y. et al. Strange Metal in Magic-Angle Graphene with near Planckian Dissipation. Phys. Rev. Lett. 124, 076801, (2020).

Bruin, J. A. N., Sakai, H., Perry, R. S. \& Mackenzie, A. P. Similarity of Scattering Rates in Metals Showing T-Linear Resistivity. Science 339, 804-807, (2013).

Cao, Y. et al. Tunable correlated states and spin-polarized phases in twisted bilayerbilayer graphene. Nature 583, 215-220, (2020).

Liu, X. et al. Tunable spin-polarized correlated states in twisted double bilayer graphene. Nature 583, 221-225, (2020).

Shen, C. et al. Correlated states in twisted double bilayer graphene. Nat. Phy. 16, 520-525, (2020).

Burg, G. W. et al. Correlated Insulating States in Twisted Double Bilayer Graphene. Phys. Rev. Lett. 123, 197702, (2019).

26 Sharma, G. et al. Carrier transport theory for twisted bilayer graphene in the metallic regime. arXiv:2003.00018 (2020).

Saito, Y., Ge, J., Watanabe, K., Taniguchi, T. \& Young, A. F. Independent superconductors and correlated insulators in twisted bilayer graphene. Nat. Phy. 16, 926-930, (2020).

Li, X., Wu, F. \& Sarma, S. D. Phonon scattering induced carrier resistivity in twisted doublebilayer graphene. Phy. Rev. B 101, 245436, (2020).

Wallbank, J. R. et al. Excess resistivity in graphene superlattices caused by umklapp electron-electron scattering. Nat. Phy. 15, 32-36, (2018).

$30 \mathrm{He}$, M. et al. Symmetry breaking in twisted double bilayer graphene. Nat. Phy. 17, 26-30, (2020).

Chen, S. et al. Electrically tunable correlated and topological states in twisted monolayerbilayer graphene. Nat. Phy., (2020).

Polshyn, H. et al. Electrical switching of magnetic order in an orbital Chern insulator. Nature, (2020). 
bilayer graphene. Nat. Phy., (2021).

34 Chen, G. et al. Evidence of a gate-tunable Mott insulator in a trilayer graphene moire superlattice. Nat. Phy. 15, 237-241, (2019). 


\section{Methods}

Flakes of graphene are exfoliated from highly oriented pyrolytic graphite, whose number of layers is determined by optical contrast. Thermal annealing is performed to ensure the stacking order of bilayer graphene to be Bernal stacking and remove contaminations on exfoliated graphene and hBN surfaces. Samples are assembled by 'cut-and-stack' method technique using Poly Bisphenol A carbonate film coating on a polydimethyl siloxane stamp placed on a glass slide. The twist angle is controlled by a rotator stage with accuracy $0.02^{\circ}$. After the pick-up procedure, the obtained heterostructure is transferred onto silicon slice with $300 \mathrm{~nm}$ silicon dioxide. Heterostructures are fabricated into Hall bar devices through electron beam lithography and reactive ion etching with $\mathrm{CHF}_{3} / \mathrm{O}_{2}$ as reactive gas, and contacted by $\mathrm{Cr} / \mathrm{Au}$ through electron beam evaporation system. Heavily doped silicon or few layer graphite are used as bottom gate and $\mathrm{Ti} / \mathrm{Au}$ metal film as top gate, respectively.

Devices are measured in a helium-4 vapor flow cryostat $(1.7 \mathrm{~K})$ with a standard lock-in technique. Typical a.c. current is applied by Stanford SR830 with a low frequency of $30.9 \mathrm{~Hz}$ and a current of $10 \mathrm{nA}$. Temperature is controlled by Cryo Con 32B PID temperature controller with long enough delay time to ensure thermal equilibrium in the device.

T-range linear fit is done by utilizing 'ployfit' function in numpy library of Python. The temperature range for linear fit is checked manually from R-T curves to avoid artifacts owning to insensitivity of the linear correlation coefficient $\mathbf{r}$. Local numerical differential of R-T plots is done by Lagrange numerical differential formula programed on Python.

\section{Figure captions}

Figure 1. Schematics of T-linear resistivity originated from (a) electron-phonon scattering and (b) quantum critical point (QCP). The Bloch-Grüneisen temperature $\left(\mathrm{T}_{\mathrm{BG}}\right)$ is defined when Fermi momentum $\mathrm{k}_{\mathrm{F}}$ and maximum phonon wave vector $\mathrm{q}$ are equal, and $\alpha>1$ is a power law coefficient when $\mathrm{T}<\mathrm{T}_{\mathrm{BG}}$.

Figure 2. T-linear resistivity and field-tunable electron-phonon interaction in $1.55^{\circ}$ TDBG. (a) Resistivity (R) as a function of carrier density (n) and displacement field (D) at $\mathrm{T}=1.8 \mathrm{~K}$. Charge neutral point, single-particle superlattice gaps on electron and hole branches are marked by CNP, $+n_{s}$, and $-n_{s}$, respectively. (b) Line cuts of $\mathrm{R}(\mathrm{T})$ at $v=2$ for $D / \varepsilon_{0}$ from 0 to $-0.7 \mathrm{~V} / \mathrm{nm}$, with a step of $0.1 \mathrm{~V} / \mathrm{nm}$. (c) Line cuts of $\mathrm{R}(\mathrm{T})$ at $D / \varepsilon_{0}=0 \mathrm{~V} / \mathrm{nm}$ for $v$ from 0.5 to 2.5 , with a step of 0.5 . The positions of these R-T curves in (b) and (c) are marked by correspondingly colored dots in (a). $\varepsilon_{0}$ is the vacuum permittivity. (d) Extracted $\mathrm{dR} / \mathrm{dT}$ as a function of $v$ in $1.55^{\circ}$ and $1.91^{\circ}$ devices. Data from $1.55^{\circ}$ and $1.91^{\circ}$ device is colored by dark red and light blue, respectively. (e) Extracted $\mathrm{dR} / \mathrm{dT}$ as a function of displacement field at $v=0.5,1,1.5$ and 2 . The light 
blue dash arrow illustrates the shift of $\mathrm{dR} / \mathrm{dT}$ peak with decreasing $v$. (f) Zoom-in plot of a, with black dash lines mark the weak cross structure and yellow dots mark the position where $\mathrm{dR} / \mathrm{dT}$ maximizes.

Figure 3. T-linear resistivity and field-driven critical point in $1.23^{\circ}$ TDBG. (a) a mapping of $R(n, D)$ at $T=1.7 \mathrm{~K}$. (b-c) Line cuts of $R(T)$ at $v=2$ (b) and $v=1.8$ (c) with $\mathrm{D} / \varepsilon_{0}$ changes from -0.1 to $-0.6 \mathrm{~V} / \mathrm{nm}$, marked by the colored dots in $\mathbf{d}$ and $\mathbf{e}$, respectively. The curves in (b) and (c) are offset in stack format for clarity and, the colored dash lines are served as guide lines. (d-e) Mappings of $\mathrm{R}(\mathrm{D}, \mathrm{T})$ at $v=2(\mathbf{d})$ and $\mathrm{v}=1.8(\mathbf{e})$. 'CS','M' and 'SBS' refer to correlated insulating states, conventional metal, symmetry broken states, respectively. The temperature boundary of SBS is determined by Fig. S10 and resistivity drop in R-T plots jointly. (f-g) Local numerical dR/dT map for (d) and (e), respectively. The critical points are marked by black arrows.

Figure 4. Correlation between $\mathrm{dR} / \mathrm{dT}$ peak and critical points of symmetry broken states in $1.23^{\circ}$ TDBG. (a) $\mathrm{dR} / \mathrm{dT}$ as a function of $\mathrm{D}$ at $\mathrm{v}=1.16,1.68$ and 2 . The curves are offset with $45 \Omega / \mathrm{K}$ for clarity. The black arrow marks the emergent $\mathrm{dR} / \mathrm{dT}$ peak at $\mathrm{v}=2$. (b) A comparison between $\mathrm{dR} / \mathrm{dT}$ and $\mathrm{R}$ at $\mathrm{v}=2$, where $\mathrm{R}$ is measured at $\mathrm{T}=1.7 \mathrm{~K}$. These vertical dash lines mark the position of $\mathrm{dR} / \mathrm{dT}$ peaks, also fits well with the critical points between conventional metal (labelled by ' $\mathrm{M}$ ') and correlated insulating states (labelled by 'I'). (c) Zoom-in of the resistivity as a function of $v$ and $D / \varepsilon_{0}$ in $1.23^{\circ}$ device. Yellow (black) dots mark the $\mathrm{dR} / \mathrm{dT}$ peak position at high (low) displacement field. (d) A comparison between $\mathrm{dR} / \mathrm{dT}$ and Hall coefficient at $\mathrm{v}=1.8$. Hall signals are measured at $\mathrm{T}=1.7 \mathrm{~K}$ with a magnetic field of $\mathrm{B}=0.1 \mathrm{~T}$ to minimize any influence on zero-field band structure. These vertical dash lines mark the positions of $\mathrm{dR} / \mathrm{dT}$ peaks, also fits well with the critical points between conventional metal (labelled by 'M') and symmetry broken states (labelled by 'SBS'). 
Figures

(a)

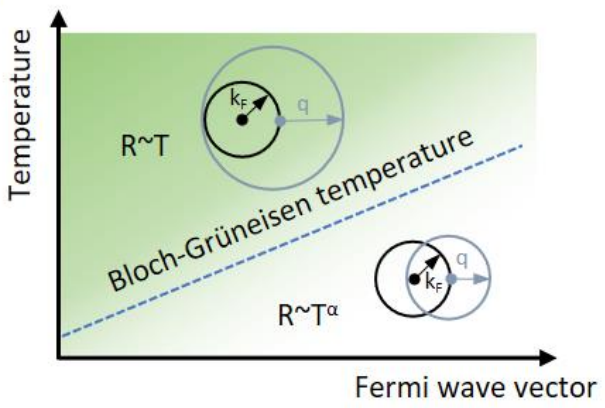

(b)

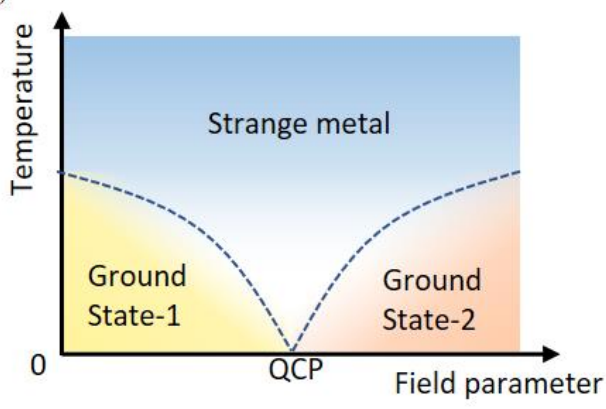

Figure 1. Schematics of T-linear resistivity originated from (a) electron-phonon scattering and (b) quantum critical point (QCP).

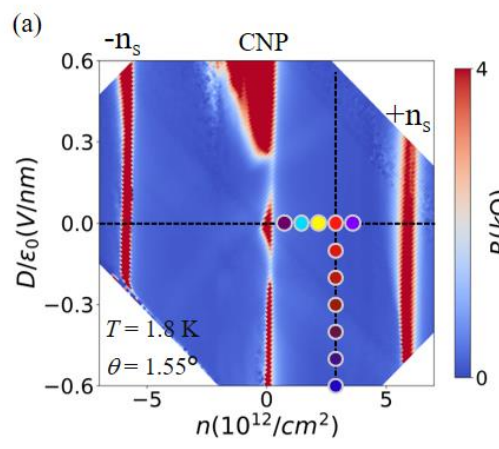

(d)

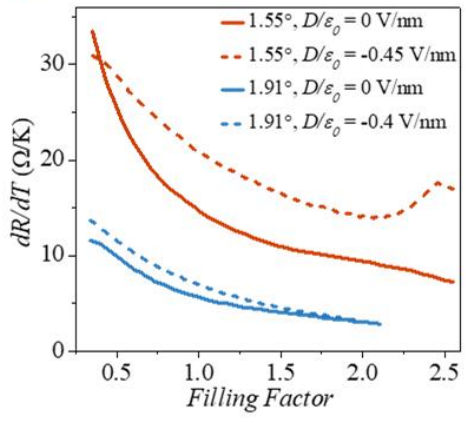

(b)

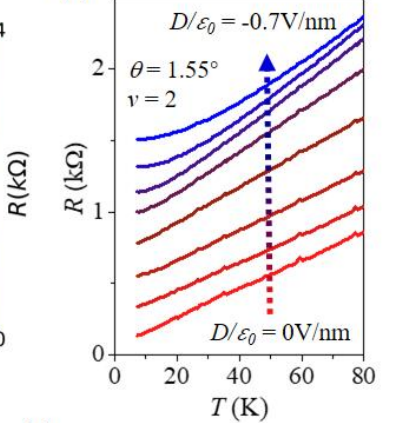

(e)

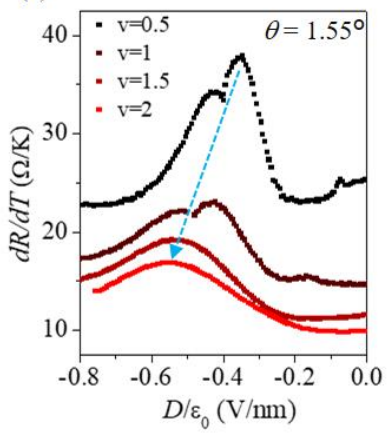

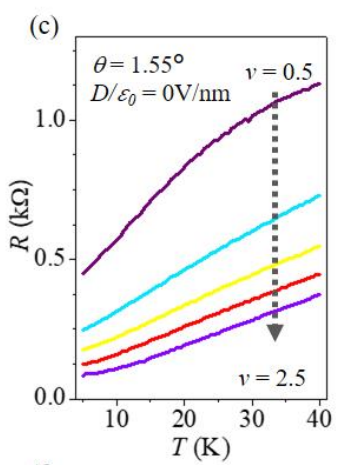

(f)

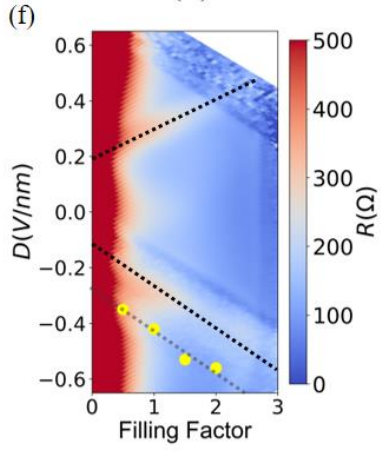

Figure 2. T-linear resistivity and field-tunable electron-phonon interaction in $1.55^{\circ}$ TDBG. 


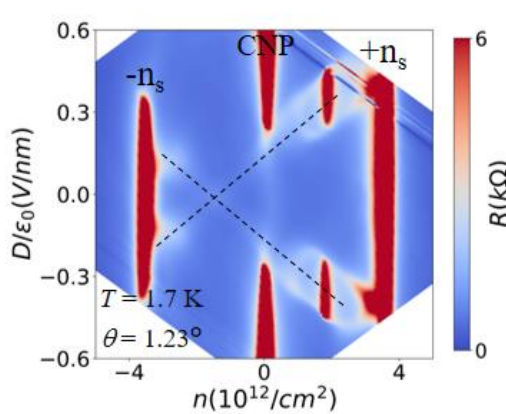

(d)

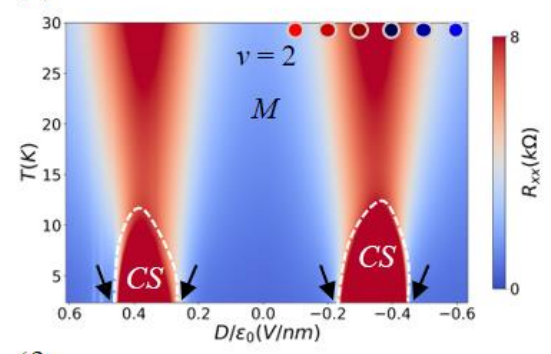

(f)

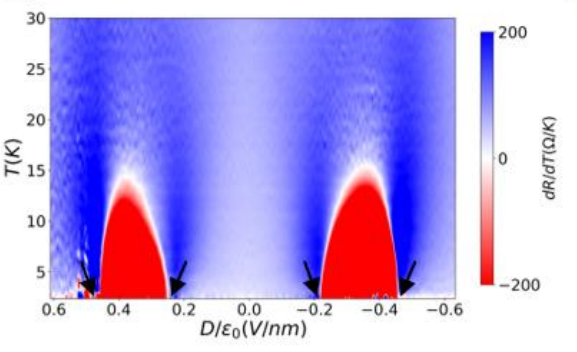

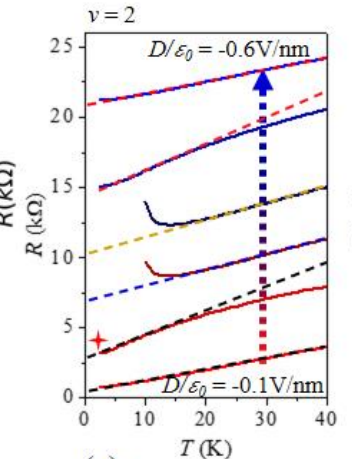

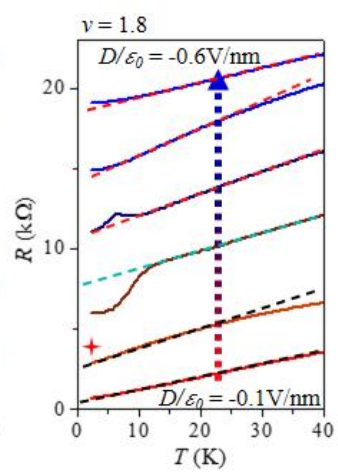

(e)

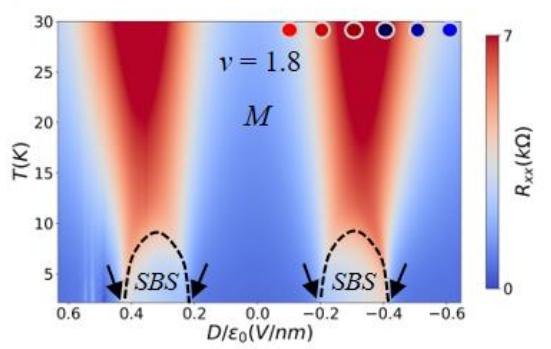

(g)

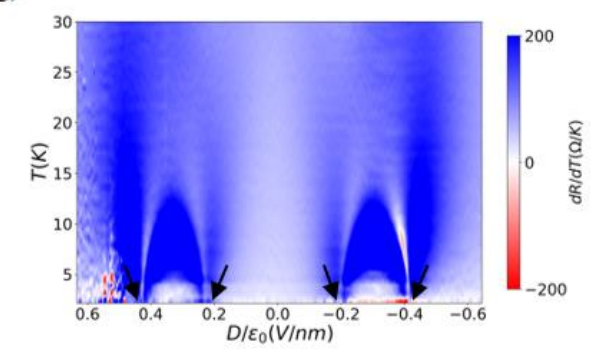

Figure 3. T-linear resistivity and field-driven critical point in $1.23^{\circ}$ TDBG.

(a)

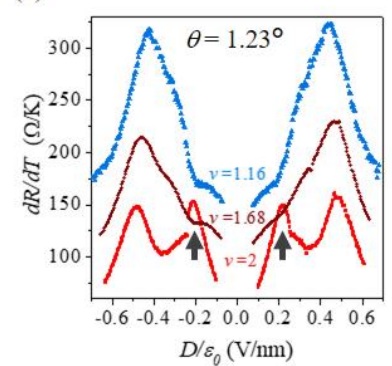

(c)

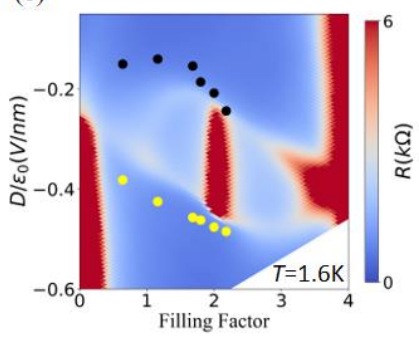

(b)

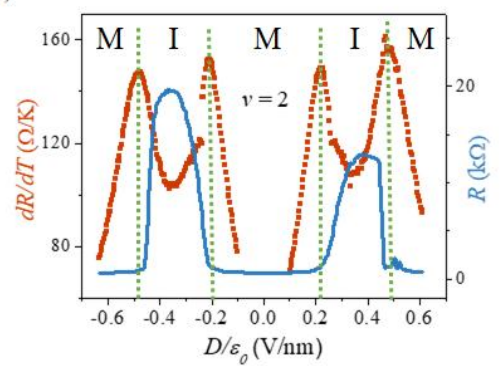

(d)

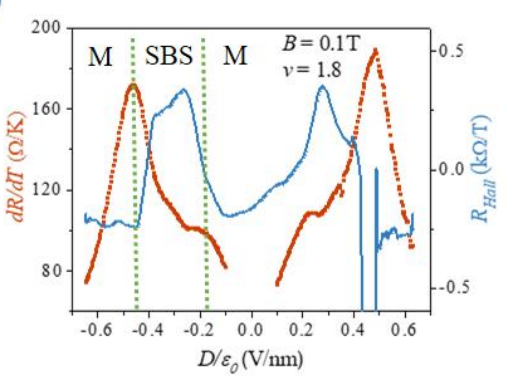

Figure 4. Correlation between critical points related $\mathrm{dR} / \mathrm{dT}$ peak and symmetry broken states in $1.23^{\circ}$ TDBG. 
(a)

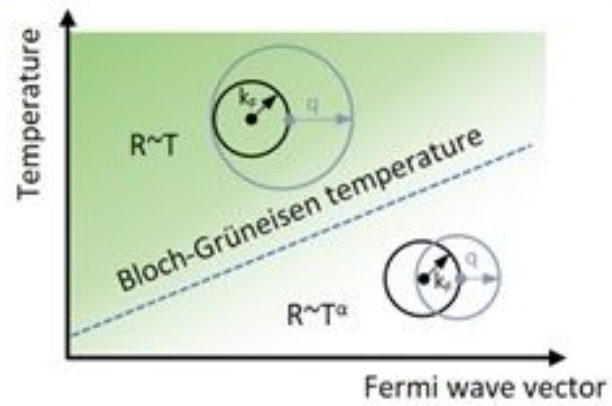

(b)

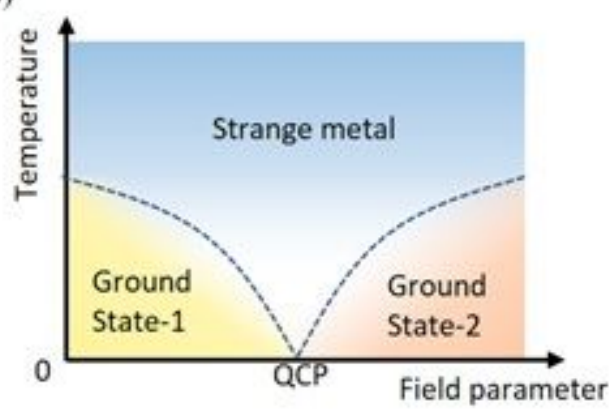

Figure 1

Schematics of T-linear resistivity originated from (a) electron-phonon scattering and (b) quantum critical point (QCP). The Bloch-Grüneisen temperature (TBG) is defined when Fermi momentum kF and maximum phonon wave vector $q$ are equal, and $a>1$ is a power law coefficient when $T<T B G$.

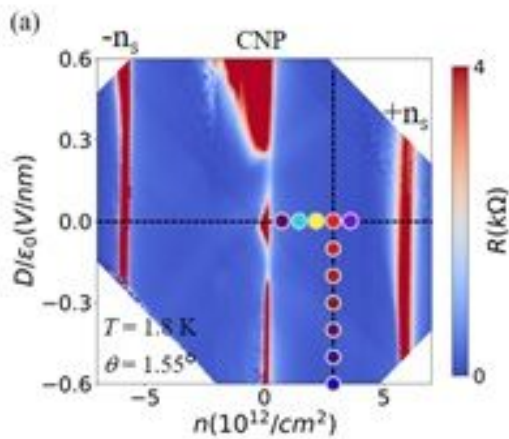

(d)

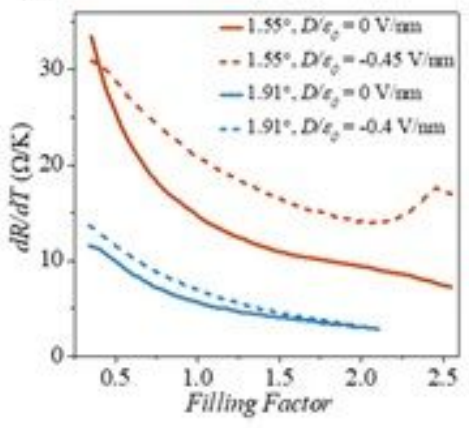

(b)

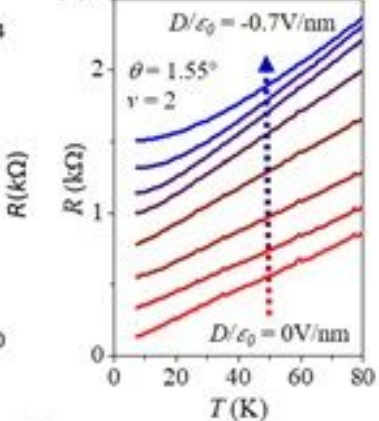

(e)

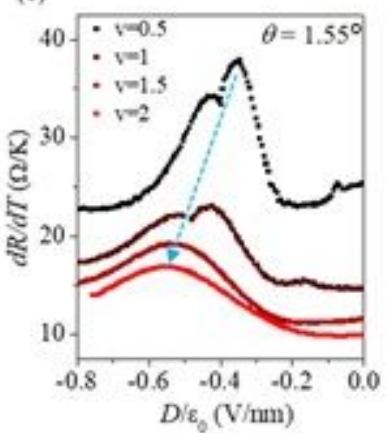

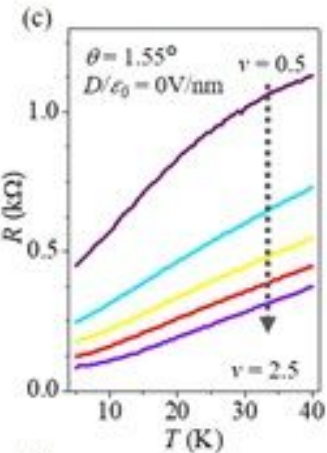

(f)

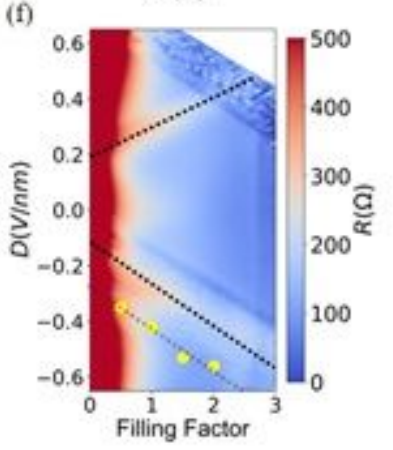

Figure 2

T-linear resistivity and field-tunable electron-phonon interaction in $1.55^{\circ}$ TDBG. (a) Resistivity (R) as a function of carrier density (n) and displacement field (D) at $T=1.8 \mathrm{~K}$. Charge neutral point, single-particle superlattice gaps on electron and hole branches are marked by CNP, $+n s$, and $-n s$, respectively. (b) Line cuts of $R(T)$ at $v=2$ for $D / e 0$ from 0 to $-0.7 \mathrm{~V} / \mathrm{nm}$, with a step of $0.1 \mathrm{~V} / \mathrm{nm}$. (c) Line cuts of $R(T)$ at $\mathrm{D} / \mathrm{e} 0=0 \mathrm{~V} / \mathrm{nm}$ for $\mathrm{v}$ from 0.5 to 2.5 , with a step of 0.5 . The positions of these R-T curves in (b) and (c) are marked by correspondingly colored dots in (a). is the vacuum permittivity. (d) Extracted dR/dT as a 
function of $v$ in $1.55^{\circ}$ and $1.91^{\circ}$ devices. Data from $1.55^{\circ}$ and $1.91^{\circ}$ device is colored by dark red and light blue, respectively. (e) Extracted $\mathrm{dR} / \mathrm{dT}$ as a function of displacement field at $\mathrm{v}=0.5,1,1.5$ and 2 . The light blue dash arrow illustrates the shift of $d R / d T$ peak with decreasing v. (f) Zoom-in plot of a, with black dash lines mark the weak cross structure and yellow dots mark the position where $\mathrm{dR} / \mathrm{dT}$ maximizes.

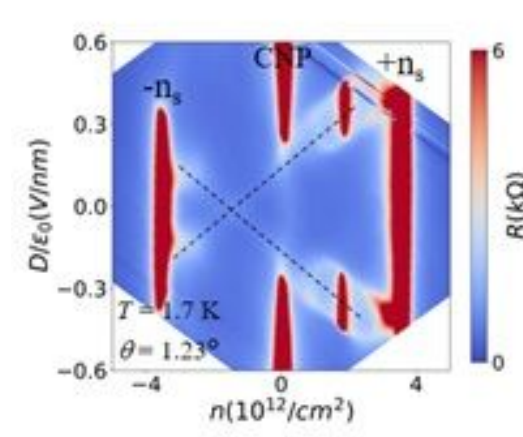

(d)

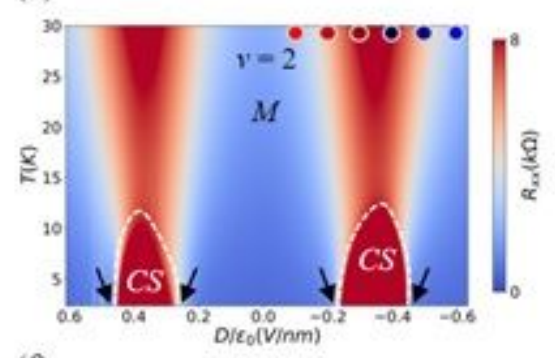

(f)

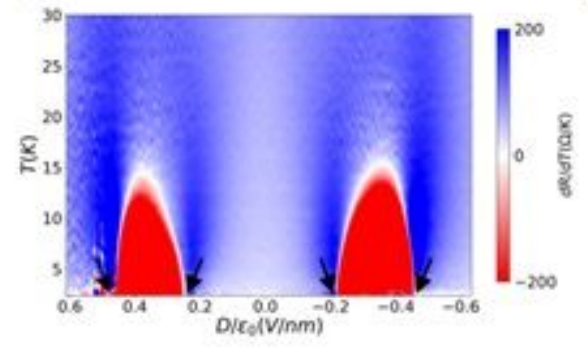

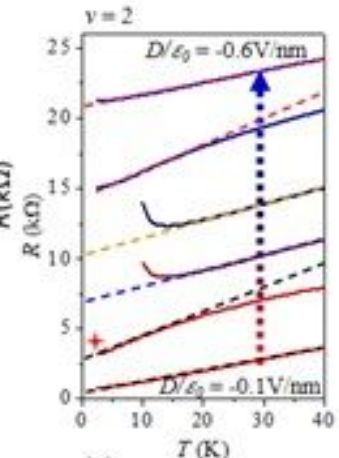

(e)

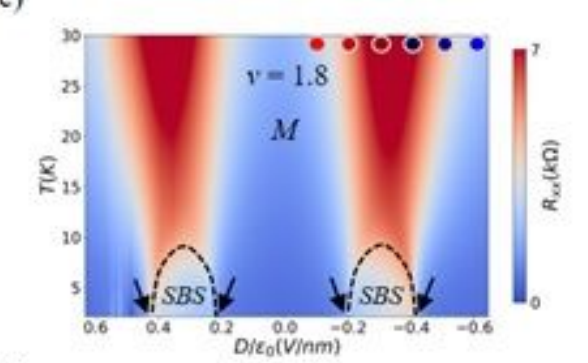

(g)

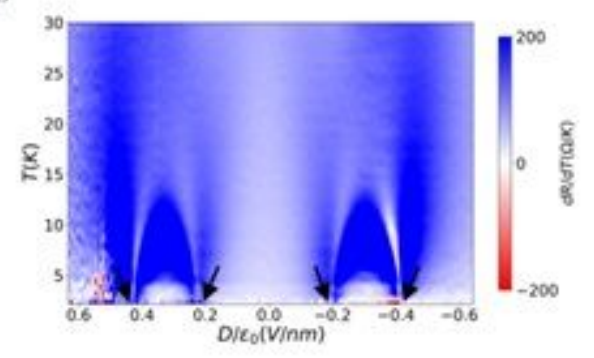

\section{Figure 3}

T-linear resistivity and field-driven critical point in $1.23^{\circ}$ TDBG. (a) a mapping of $R(n, D)$ at $T=1.7 K$. (b-c) Line cuts of $R(T)$ at $v=2$ (b) and $v=1.8$ (c) with $D / e 0$ changes from -0.1 to $-0.6 \mathrm{~V} / \mathrm{nm}$, marked by the colored dots in $d$ and e, respectively. The curves in (b) and (c) are offset in stack format for clarity and, the colored dash lines are served as guide lines. (d-e) Mappings of $R(D, T)$ at $v=2(d)$ and $v=1.8(e)$. 'CS', $M$ ' and 'SBS' refer to correlated insulating states, conventional metal, symmetry broken states, respectively. The temperature boundary of SBS is determined by Fig. S10 and resistivity drop in R-T plots jointly. (f-g) Local numerical dR/dT map for (d) and (e), respectively. The critical points are marked by black arrows. 
(a)

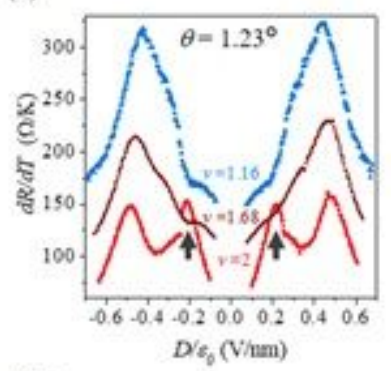

(c)

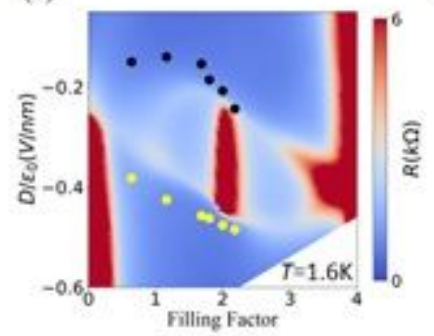

(b)

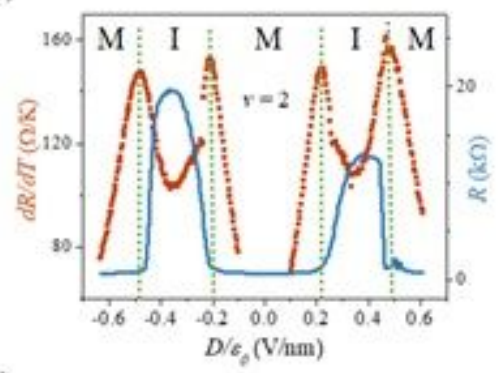

(d)

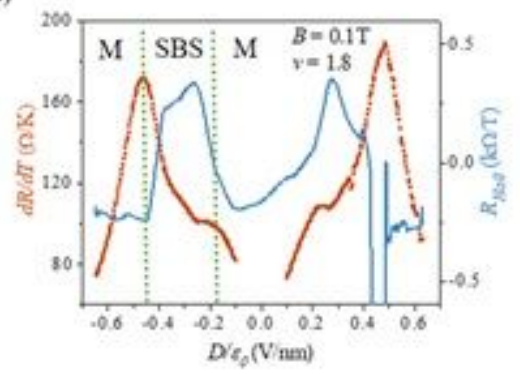

Figure 4

Correlation between dR/dT peak and critical points of symmetry broken states in $1.23^{\circ}$ TDBG. (a) dR/dT as a function of $D$ at $v=1.16,1.68$ and 2 . The curves are offset with $45 \Omega / K$ for clarity. The black arrow marks the emergent $d R / d T$ peak at $v=2$. (b) A comparison between $d R / d T$ and $R$ at $v=2$, where $R$ is measured at $T=1.7 \mathrm{~K}$. These vertical dash lines mark the position of $d R / d T$ peaks, also fits well with the critical points between conventional metal (labelled by ' $\mathrm{M}$ ') and correlated insulating states (labelled by ' I'). (c) Zoom-in of the resistivity as a function of $\mathrm{v}$ and $\mathrm{D} / \mathrm{e} 0$ in $1.23^{\circ}$ device. Yellow (black) dots mark the $\mathrm{dR} / \mathrm{dT}$ peak position at high (low) displacement field. (d) A comparison between $\mathrm{dR} / \mathrm{dT}$ and Hall coefficient at $v=1.8$. Hall signals are measured at $T=1.7 \mathrm{~K}$ with a magnetic field of $B=0.1 \mathrm{~T}$ to minimize any influence on zero-field band structure. These vertical dash lines mark the positions of $\mathrm{dR} / \mathrm{dT}$ peaks, also fits well with the critical points between conventional metal (labelled by ' $\mathrm{M}$ ') and symmetry broken states (labelled by 'SBS').

\section{Supplementary Files}

This is a list of supplementary files associated with this preprint. Click to download.

- RTTDBGsupplementary210412.docx 